\title{
PSEUDO LATTICE PROPERTIES OF THE STAR-ORTHOGONAL PARTIAL ORDERING FOR STAR-REGULAR RINGS
}

\author{
ROBERT E. HARTWIG
}

\begin{abstract}
It is shown that a star-regular ring $\boldsymbol{R}$ forms a pseudo upper semilattice under the star-orthogonal partial ordering. That is, for every $a, b$ in $R$, the set $\{c \mid c>a, c>b\}$ is nonempty if and only if $a \vee b$ exists in $R$, in which case
\end{abstract}

$$
a \vee b=a+\left(1-a a^{\dagger}\right) b b^{*}\left[\left(1-a^{\dagger} a\right) b^{*}\right]^{\dagger}
$$

1. Introduction. In two recent papers ([2], [3]), Drazin introduced the star-orthogonal partial ordering

$$
a<b \Leftrightarrow a^{*} a=a^{*} b \text { and } a a^{*}=b a^{*}
$$

for proper-star-semigroups $\left(S,{ }^{*}\right)$, for which the involution $(\cdot)^{*}: S \rightarrow S$ satisfies, in addition to the usual two conditions (i) $\left(a^{*}\right)^{*}=a$, (ii) $(a b)^{*}=b^{*} a^{*}$, the "proper" condition (iii) $a^{*} a=a^{*} b=b^{*} a=b^{*} b \Rightarrow a=b$. For a ring $R$, the condition (iv) $(a+b)^{*}=a^{*}+b^{*}$ is added, and (iii) is easily seen to be equivalent to the traditional star cancellation law

$$
a^{*} a=0 \Rightarrow a=0 \text {. }
$$

It was subsequently shown by Hartwig and Drazin [6] that the algebra $\mathbf{C}_{n \times n}$ of $n \times n$ complex matrices forms a lower semilattice under the partial ordering (1), which means that $a \wedge b=\sup \{c \mid c<a, c<b\}$ exists in $\mathbf{C}_{n \times n}$ for all $a, b$ in $\mathbf{C}_{n \times n}$. Because invertible elements are obviously maximal elements under $<$, the join $a \bigvee b=\inf \{c \mid c>a, c>b\}$ will in general not exist, because the set $\{c \mid c>a, c>b\}$ may be empty.

The purpose of this note is to prove that if $R$ is a star-regular ring, then $R$ forms a pseudo upper semilattice, that is $a \bigvee b$ will exist precisely when $\{c \mid c>a, c>b\}$ is nonempty. An element $a \in S$ is called regular if $a \in a S a$, and *-regular if $a a^{*}$ and $a^{*} a$ are both regular. It is well known, from [8], that $a \in S$ is star-regular exactly when there is a, necessarily unique, solution to the equations:

$$
a x a=a, \quad x a x=x, \quad(a x)^{*}=a x, \quad(x a)^{*}=x a .
$$

This solution $a^{\dagger}$ is known as the Moore-Penrose inverse of $a$. A ring is called (star) regular when every element $a \in R$ is (star) regular. It should be noted that $R$ is *-regular precisely when $R$ is regular and the involution is proper.

Received by the editors February 15, 1978 and, in revised form, November 21, 1978.

AMS (MOS) subject classifications (1970). Primary 06A10, 06A20; Secondary 15A28, $15 A 30$.

(C) 1979 American Mathematical Society 0002-9939/79/0000-0550/\$02.25 
2. Main results. We shall now prove our main local result, from which the global result for star-regular rings obviously follows.

THEOREM 1. Let $R$ be a ring with involution $(\cdot)^{*}$, and let $a, b$ be elements of $R$ such that $a, b,\left(1-a a^{\dagger}\right) b$, and $b\left(1-a^{\dagger} a\right)$ are star-regular. Then $\{c \mid c>a$, $c>b$ \} is nonempty if and only if

$$
\begin{aligned}
& \text { (i) } b\left(b^{*}-a^{*}\right) a=0=a\left(b^{*}-a^{*}\right) b, \\
& \text { (ii) } b\left(b^{*}-a^{*}\right) \in b\left(1-a^{\dagger} a\right) R, \\
& \text { (iii) }\left(b^{*}-a^{*}\right) b \in R\left(1-a a^{\dagger}\right) b .
\end{aligned}
$$

In which case $a \vee b$ exists and is given by

$$
a \vee b=a+\left(1-a a^{\dagger}\right) b b^{*}\left[\left(1-a^{\dagger} a\right) b^{*}\right]^{\dagger}
$$

Proof. Suppose that $c \geqslant a, c \geqslant b$ for some $c \in R$, that is

$$
a^{*}(a-c)=(a-c) a^{*}=b^{*}(b-c)=(b-c) b^{*}=0 .
$$

Since $a$ and $b$ are *-regular, $a^{\dagger}$ and $b^{\dagger}$ exist and hence (5a) may be rewritten as in [3]:

$$
a a^{\dagger} c=a=c a^{\dagger} a, \quad b b^{\dagger} c=b=c c^{\dagger} b
$$

Thus

$$
a^{\dagger} b=a^{\dagger} c b^{\dagger} b=a^{\dagger}\left(a a^{\dagger} c b^{\dagger} b\right)=a^{\dagger} a b^{\dagger} b, \quad b^{\dagger} a=b^{\dagger} b a^{\dagger} a .
$$

Symmetry now yields two more such results. From (6), $a^{*} b=a^{*} a b^{\dagger} b$, which shows that $b^{\dagger} b a^{*} a=b^{*} a$ and hence that $b a^{*} a=b b^{*} a$. By symmetry $a a^{*} b=$ $a b^{*} b$, so that (3i) follows. Next, let $u=\left(1-a a^{\dagger}\right) b$ and $v=b\left(1-a^{\dagger} a\right)$, and consider $b^{*} c=b^{*} b$. Post multiplication by $\left(1-a^{\dagger} a\right)$ yields

$$
b^{*} b\left(1-a^{\dagger} a\right)=b^{*} c\left(1-a^{\dagger} a\right)=b^{*}\left(1-a a^{\dagger}\right) c
$$

that is

$$
u^{*} c=b^{*} v \text {. }
$$

Similarly $\left(1-a a^{\dagger}\right) c b^{*}=c\left(1-a^{\dagger} a\right) b^{*}=\left(1-a a^{\dagger}\right) b b^{*}$ yields

$$
c v^{*}=u b^{*} \text {. }
$$

The assumed consistency of (7) and (8) ensures that $u^{*} u^{* \dagger} b^{*} v=b^{*} v$ and $u b^{*} v^{* \dagger} v^{*}=u b^{*}$, while the elimination of $c$ gives

$$
u^{*} u b=u^{*} c v^{*}=b^{*} v v^{*} \text {. }
$$

Now

$$
u^{\dagger} u b^{*} v=b^{*} v \Leftrightarrow v^{*} b=v^{*} b u^{\dagger} u \Leftrightarrow v^{*} b \in R u \Leftrightarrow v^{\dagger} b=v^{\dagger} b u^{\dagger} u,
$$

where

$$
v^{*} b=\left(1-a^{\dagger} a\right) b^{*} b=b^{*} b-a^{\dagger} a b^{*} b=b^{*} b-a^{*} b b^{\dagger} b=\left(b^{*}-a^{*}\right) b,
$$

from which (3iii) follows.

Similarly

$$
u b^{*} v v^{\dagger}=u b^{*} \Leftrightarrow v v^{\dagger} b u^{*}=b u^{*} \Leftrightarrow b u^{*} \in v R \Leftrightarrow v v^{\dagger} b u^{\dagger}=b u^{\dagger},
$$


where $b u^{*}=b b^{*}\left(1-a a^{\dagger}\right)=b b^{*}-b a^{*}$. This completes the proof of the necessity of (3).

Suppose now that (3i), (3ii) and (3iii) hold. We shall first demonstrate that $\{r \mid r>a, r>b\}$ is nonempty.

First observe that a particular solution to the equations (7) and (8) alone is given by $u^{* \dagger} b^{*} v$. To obtain a solution to $r \geqslant a, r \geqslant b$, all we have to do is add element $a$ to $w=u^{* \dagger} b^{*} v$.

Indeed, since $a^{\dagger} u=0=u^{\dagger} a=v a^{\dagger}=a v^{\dagger}$, we have $a^{\dagger} w=w a^{\dagger}=0$, and

$$
a a^{\dagger}(a+w)=a=(a+w) a^{\dagger} a \text { or } a<a+w .
$$

Next, consider $b b^{\dagger}(a+w)=b a^{\dagger} a+b b^{\dagger} u^{* \dagger} b^{*} v$, where we used (3i), and recall that always:

$$
\begin{array}{lll}
u^{*} u=u^{*} b=b^{*} u, & v v^{*}=v b^{*}=b v^{*}, & u b^{\dagger} b=u, \\
u^{\dagger} u=u^{\dagger} b, & v v^{\dagger}=b v^{\dagger}, & b b^{\dagger} v=v, \\
u=u u^{\dagger} b, & v=b v^{\dagger} v . &
\end{array}
$$

Hence, $b b^{\dagger} u^{* \dagger} b^{*} v=\left(u^{\dagger} b b^{\dagger}\right)^{*} b^{*} v$ which by (11) becomes $\left(u^{\dagger} u b^{\dagger}\right)^{*} b^{*} v=$ $b^{* \dagger}\left(u^{\dagger} u b^{*} v\right)$. Using (10a) this reduces to $b^{* \dagger} b^{*} v=b b^{\dagger} v$, and hence by (11) equals $v=b-b a^{\dagger} a$. Substituting this in the above we see that $b b^{\dagger}(a+w)=$ b. Similarly, with aid of (10b), (3i) and (11), $(a+w) b^{\dagger} b=a a^{\dagger} b+u^{* \dagger} b^{*} v b^{\dagger} b$ $=a a^{\dagger} b+u=b$, and thus $a \leqslant a+w, b \leqslant a+w$, as desired. In conclusion let us prove that $a+w$ is in fact equal to $a \vee b$. In order to do this, let us first verify that $w^{\dagger}$ exists and that

$$
(a+w)^{\dagger}=a^{\dagger}+w^{\dagger}
$$

The details are essential since we shall also need the expressions for $(a+w)(a+w)^{\dagger}$ and $(a+w)^{\dagger}(a+w)$. Again, since $a^{*} w=0=w a^{*}$, it follows by a result of Hestenes [7] that (12) holds and that in addition:

$$
(a+w)(a+w)^{\dagger}=a a^{\dagger}+w w^{\dagger}, \quad(a+w)^{\dagger}(a+w)=a^{\dagger} a+w^{\dagger} w,
$$

provided $w^{\dagger}$ exists. Let us now verify that $x=v^{\dagger} b^{* \dagger} u^{*}=w^{\dagger}$.

Indeed, $w x=u^{* \dagger} b^{*} v v^{\dagger} b^{* \dagger} u^{*}=\left(v v^{\dagger} b u^{\dagger}\right)^{*} b^{* \dagger} u^{*}$, which by (10b) becomes

$$
\left(b u^{\dagger}\right)^{*} b^{* \dagger} u^{*}=u^{* \dagger} b^{*} b^{* \dagger} u^{*}=u^{* \dagger} b^{\dagger} b u^{*}=\left[\left(u b^{\dagger} b\right) u^{\dagger}\right]^{*} \text {. }
$$

But $u b^{\dagger} b=u$, and hence we arrive at $w x=\left(u u^{\dagger}\right)^{*}=u u^{\dagger}$.

Similarly $x w=v^{\dagger} b^{* \dagger} u^{*} u^{* \dagger} b^{*} v=v^{\dagger} b^{* \dagger} u^{\dagger} u b^{*} v$, which by (10a) reduces to

$$
v^{\dagger} b^{* \dagger} b^{*} v=v^{\dagger} b b^{\dagger} v=v^{\dagger} v \text {, since } b b^{\dagger} v=v \text {. }
$$

Hence, $w x w=u u^{\dagger} w=w$ and $x w x=v^{\dagger} v\left(v^{\dagger} b^{* \dagger} u^{*}\right)=x$, as desired. Consequently, we may conclude that

$$
(a+w)(a+w)^{\dagger}=a a^{\dagger}+u u^{\dagger},(a+w)^{\dagger}(a+w)=a^{\dagger} a+v^{\dagger} v .
$$

Finally let $c \geqslant a, c \geqslant b$, so that (5) holds. Then $(a+w)(a+w)^{\dagger} c=\left(a a^{\dagger}\right.$ $\left.+u u^{\dagger}\right) c=a+u u^{\dagger} c=a+w$, since $u u^{\dagger} c=u^{*} u^{*} c=u^{* \dagger} b^{*} v$. Similarly $c(a$ $+w)^{\dagger}(a+w)=c\left(a^{\dagger} a+v^{\dagger} v\right)=a+c v^{\dagger} v$ in which $c v^{\dagger} v=c v^{*} v^{* \dagger}$. Using (8) this equals $u b^{*} v^{* \dagger}=u^{* \dagger}\left(u^{*} u b^{*}\right) v^{* \dagger}$ and hence yields, with aid of (9), 
$u^{* \dagger}\left(b^{*} v v^{*}\right) v^{* \dagger}=u^{* \dagger} b^{*} v=x$. Thus $a+w<c$ and consequently $a \vee b=a$ $+u^{* \dagger} b^{*} v=a+u b^{*} v^{* \dagger}$.

3. Remarks and conclusions. Let us conclude this note with several remarks and conclusions.

(i) For projections (or Hermitian idempotents), $e$ and $f$, the conditions (3) automatically hold because obviously $e(f-e) f=0=f(f-e) e, f(f-e)=$ $f(1-e)$, and $(f-e) f=(1-e) f$. Thus

$$
e \vee f=e+(1-e) f[(1-e) f]^{\dagger}=e+(1-e)[(1-e) f]^{\dagger},
$$

which is well known [1], [6].

(ii) When $a$ and $b$ star-commute, that is when $a^{*} b$ and $b a^{*}$ are Hermitian, then (3ii) and (3iii) hold automatically. To prove this we begin by observing that $a a^{*}$ and $b b^{*}$ commute. Since $\left(a a^{*}\right)^{\dagger}$ is the group inverse of $a a^{*}$, it follows by a result of Drazin $\left[4\right.$, p. 208], that $\left(a a^{*}\right)^{\dagger}$ and $b b^{*}$ also commute. Next, we note that

$$
a^{\dagger} b b^{*}=a^{*}\left(a a^{*}\right)^{\dagger} b b^{*}=a^{*} b b^{*}\left(a a^{*}\right)^{\dagger}=b^{*} b a^{*}\left(a a^{*}\right)^{\dagger}=b^{*} b a^{\dagger} .
$$

Lastly, we need the fact that $\left(b^{*} a\right)^{\dagger}=a^{\dagger} b^{* \dagger}$ and $\left(a^{*} b\right)^{\dagger}=b^{\dagger} a^{* \dagger}$, which may be verified directly or by using the reverse order law [5, p. 231]. Combining these see that $a^{\dagger} b=\left(a^{\dagger} b b^{*}\right) b^{* \dagger}=b^{*} b a^{\dagger} b^{* \dagger}=b^{*} b\left(b^{*} a\right)^{\dagger}=b^{*} b\left(a^{*} b\right)^{\dagger}=$ $b^{*} b b^{\dagger} a^{* \dagger}=b^{*} a^{* \dagger}$, that is, $a^{\dagger} b$ is also Hermitian. Hence $a a^{\dagger} b=a b^{*} a^{* \dagger}=$ $b a^{*} a^{* \dagger}=b a^{\dagger} a$, which implies that $u=v$. Thus, with aid of (11) $v^{*} b=u^{*} b$ $=u^{*} u \in R u$ while $b u^{*}=b v^{*}=v v^{*} \in v R$. This means that

$$
a \vee b \text { exists } \Leftrightarrow b\left(b^{*}-a^{*}\right) a=0=a\left(b^{*}-a^{*}\right) b
$$

In which case

$$
a \bigvee b=a+u^{* \dagger} b^{*} v=a+u^{* \dagger} u^{*} u=a+\left(1-a a^{\dagger}\right) b .
$$

(iii) If $a$ and $b$ are partial isometries, such that $a^{*}=a^{\dagger}$ and $b^{*}=b^{\dagger}$, or equivalently $a a^{*} a=a, b b^{*} b=b$, then (14) also holds! The proof, however, is more delicate. First note that with aid of (3i) $u^{*} u b^{*}=b^{*} v v^{*}$. This allows us to conclude that $b u^{*}$ and $v b^{*}$ are both star-regular. Indeed,

$$
\left(b u^{*}\right)\left(b u^{*}\right)^{*}=b u^{*} u b^{*}=b b^{*} v v^{*}=b b^{*} b\left(1-a^{\dagger} a\right) v^{*}=v v^{*} \text {, }
$$

and

$$
\left(b u^{*}\right)^{*}\left(b u^{*}\right)=u b^{*} b u^{*}=\left(1-a a^{\dagger}\right) b b^{*} b u^{*}=u u^{*}
$$

Similarly,

$$
\left(v^{*} b\right)\left(v^{*} b\right)^{*}=v^{*} b b^{*} v=v^{*} b b^{*} b\left(1-a^{\dagger} a\right)=v^{*} v
$$

and

$$
\left(v^{*} b\right)^{*}\left(v^{*} b\right)=b^{*} v v^{*} b=u^{*} u b^{*} b=u^{*} u,
$$

all of which are regular by assumption. Hence

$$
b u^{*}=\left(b u^{*}\right)\left(b u^{*}\right)^{*}\left(b u^{*}\right)^{* \dagger}=v v^{*}\left(b u^{*}\right)^{* \dagger} \in v R
$$


and

$$
v^{*} b=\left(v^{*} b\right)^{* \dagger}\left(v^{*} b\right)^{*}\left(v^{*} b\right)=\left(v^{*} b\right)^{* \dagger} u^{*} u \in R u
$$

as desired.

(iv) Using (1-21) of [5] we may rewrite (4) as

$$
a \vee b=a+\left(1-a a^{\dagger}\right) b b^{*}\left[\left(1-a^{\dagger} a\right) b^{*}\right]^{\dagger}\left(1-a^{\dagger} a\right)
$$

however no $(a-b)$-symmetric formula is known at the present.

(v) Since $u u^{\dagger} c=u^{*} b^{*} v$ for all $c>a, b$, we have the following identity in $a \vee b-a, a \vee b-a=u u^{\dagger}(a \vee b-a) v^{\dagger} v$.

(vi) It is not known whether $a \vee b$ exists in a general star-regular ring, however it is anticipated that $u$ and $v$ will play a dominant role in its investigation.

\section{REFERENCES}

1. K. Berberian, Baer star-rings, Springer-Verlag, Berlin-New York, 1972.

2. M. P. Drazin, The Moore-Penrose inverse in abstract operator rings, Notices Amer. Math. Soc. 23 (1976), A-664. Abstract \# 740-B20.

3. _ Natural structures on semigroups with involution, Bull. Amer. Math. Soc. 84 (1978), 139-141.

4. __ Pseudo-inverses in associative rings and semigroups, Amer. Math. Monthly 65 (1958), 506-514.

5. R. E. Hartwig, Block generalized inverses, Arch. Rational Mech. Anal. 61 (1976), 197-251.

6. R. E. Hartwig and M. P. Drazin, Lattice properties of the star-order for complex matrices (submitted).

7. M. R. Hestenes, Relative hermitian matrices, Pacific J. Math. 11 (1961), 225-245.

8. N. S. Urquhart, Computation of generalized inverse matrices which satisfy specified conditions, SIAM Rev. 10 (1968), 216-218.

Department of Mathematics, North Carolina State University, Raleigh, North Carolina 27607 